Canad. Math. Bull. Vol. 20 (1), 1977

\title{
SOME MAPPINGS ASSOCIATED WITH THE PERMUTATION GROUPS.
}

\author{
BY \\ J. L. DAVISON
}

1. Introduction. Let $S_{n}$ denote the permutation group on $\{1,2,3, \ldots, n\}$. Let $k \geq 1$ and $n \geq 1$ be integers. For $\sigma \in S_{n}$ we define ([1]) $m_{k, n}: S_{n} \rightarrow \mathbf{Z}$ by

$$
m_{k, n}(\sigma)=\sum_{i=1}^{n}|\sigma(i)-i|^{k}
$$

Let $\rho_{n} \in S_{n}$ be the reverse permutation: that is,

$$
\rho_{n}(i)=n+1-i \quad(1 \leq i \leq n) .
$$

We define integers $f_{k, n}$ by setting $f_{k, n}=m_{k, n}\left(\rho_{n}\right)$. These integers play an important rôle in what follows, so we include a small table of values of the $f_{k, n}$.

$\begin{array}{crrrrrrrrrr}k / n & 1 & 2 & 3 & 4 & 5 & 6 & 7 & 8 & 9 & 10 \\ 1 & 0 & 2 & 4 & 8 & 12 & 18 & 24 & 32 & 40 & 50 \\ 2 & 0 & 2 & 8 & 20 & 40 & 70 & 112 & 168 & 240 & 330 \\ 3 & 0 & 2 & 16 & 56 & 144 & 306 & 576 & 992 & 1,600 & 2,450 \\ 4 & 0 & 2 & 32 & 164 & 544 & 1,414 & 3,136 & 6,216 & 11,328 & 19,338\end{array}$

Let $J_{k, n}$ denote the set of even integers in the interval $\left[0, f_{k, n}\right]$. It is proved in Theorem 1 that $m_{k, n}\left(S_{n}\right) \subseteq J_{k, n}$. In [1], it was further proved that $m_{2, n}\left(S_{n}\right)=J_{2, n}$ for $n \geq 4$. It is easy to see (Proposition 1) that $m_{1, n}\left(S_{n}\right)=J_{1, n}$ for all $n \geq 1$. Thus, it seems natural to ask whether $m_{k, n}\left(S_{n}\right)=J_{k, n}$ for each $k$ (and large enough $n$ ). The main result of this paper is to prove:

THeOREM 2. If $k \geq 3$ then for all $n \in N, f_{k, n}-4 \notin m_{k, n}\left(S_{n}\right)$.

Thus in general $m_{k, n}\left(S_{n}\right) \neq J_{k, n}$. In $\S 3$ we give a partial result on $m_{3, n}\left(S_{n}\right)$ and indicate a reasonable conjecture which we have been unable to prove as yet.

\section{Main results}

LEMMA 1. If $\sigma \in S_{n}$, then $m_{k, n}(\sigma)$ is an even integer for any $k \geq 1$.

Proof. Let $a_{i}=|\sigma(i)-i|$. Then $\sigma(i)-i= \pm a_{i}$

Let

$$
\begin{aligned}
& I_{1}=\left\{i: 1 \leq i \leq n: \sigma(i)-i=+a_{i}\right\} \\
& I_{2}=\left\{i: 1 \leq i \leq n: \sigma(i)-i=-a_{i}\right\}
\end{aligned}
$$

Received by the editors Feb. 9, 1976. 
Then

$$
\sum_{i=1}^{n}(\sigma(i)-i)=\sum_{i \in I_{1}} a_{i}-\sum_{i \in I_{2}} a_{i}
$$

But

$$
\sum_{i=1}^{n} \sigma(i)=\sum_{i=1}^{n} i \quad \text { so that } \sum_{i \in I_{1}} a_{i}=\sum_{i \in I_{2}} a_{i}
$$

Thus

$$
\sum_{i=1}^{n} a_{i}=2 \sum_{i \in I_{2}} a_{i}
$$

is even. So there is an even number of those integers $a_{i}$ which are odd and hence for any $k \geq 1 m_{k, n}(\sigma)=\sum_{i=1}^{n} a_{i}^{k}$ is even.

Remark. Equation $\left({ }^{*}\right)$ gives a condition on the permissible decompositions of an even integer into sums of $k$ th powers. For example, the integer 24 cannot belong to $m_{3,9}\left(S_{9}\right)$ since the decomposition $24=2^{3}+2^{3}+2^{3}$ would imply that $\{2,2,2\}$ can be split into two subsets with equal sums, which clearly is not the case.

THEOREM 1. For each pair of integers $k, n: m_{k, n}\left(S_{n}\right) \subseteq J_{k, n}$

Proof. Let $\sigma \in S_{n}$, and suppose $\sigma$ is not the reverse permutation. Then there exists an integer $i, 1 \leq i \leq n-1$, such that $\sigma(i)<\sigma(i+1)$. Let $\tau$ be the transposition $(i i+1)$, and $\mu=\sigma \circ \tau$. Then we show that $m_{k, n}(\mu)>m_{k, n}(\sigma)$. Since $\rho_{n}$ is obtainable from any $\sigma$ by transpositions of such a kind this will show that $m_{k, n}\left(S_{n}\right) \subseteq J_{k, n}$.

To simplify the notation, let $x=\sigma(i)-i$ and $y=\sigma(i+1)-(i+1)$. Then $x \leq y$. The proof splits into two parts, depending on whether $k$ is even or odd.

Suppose first that $k$ is even. Then it is clear that

$$
\begin{aligned}
m_{k, n}(\mu)-m_{k, n}(\sigma) & =\left\{(x-1)^{k}+(y+1)^{k}\right\}-\left\{x^{k}+y^{k}\right\} \\
& =k\left(y^{k-1}-x^{k-1}\right)+\left(\begin{array}{l}
k \\
2
\end{array}\right)\left(y^{k-2}+x^{k-2}\right)+\cdots+k(y-x)+2
\end{aligned}
$$

Now since $y \geq x$ and $k$ is even, every second term in this expansion, starting from the first, is non-negative. The other terms are clearly all non-negative also. Thus $m_{k, n}(\mu) \geq m_{k, n}(\sigma)+2$. Note also that

$$
m_{k, n}(\mu)-m_{k, n}(\sigma)=2 \Leftrightarrow x=y=0
$$

and that otherwise

$$
m_{k, n}(\mu)-m_{k, n}(\sigma) \geq\left(\begin{array}{l}
k \\
2
\end{array}\right)+2>4 \text { for } k \geq 3 .
$$


Consider now when $k$ is odd. Three cases can arise
(i) $y \geq x \geq 1$
(ii) $x \leq y \leq-1$
(iii) $x \leq 0 \leq y$

We deal with these cases separately

(i) $y \geq x \geq 1$

Here

$$
\begin{aligned}
m_{k}(\mu)-m_{k}(\sigma) & =\left\{(x-1)^{k}+(y+1)^{k}\right\}-\left\{x^{k}+y^{k}\right\} \\
& =k\left(y^{k-1}-x^{k-1}\right)+\left(\begin{array}{l}
k \\
2
\end{array}\right)\left(y^{k-2}+x^{k-2}\right)+\cdots+k(y+x)
\end{aligned}
$$

We see then that $m_{k, n}(\mu) \geq m_{k, n}(\sigma)+2 k$

(ii) $x \leq y \leq-1$. Let $u=-x$ and $v=-y$. Then $u \geq v \geq 1$ and

$$
m_{k, n}(\mu)-m_{k, n}(\sigma)=\left\{(u+1)^{k}+(v-1)^{k}\right\}-\left\{u^{k}+v^{k}\right\}
$$

which reduces to case (i).

(iii) $x \leq 0 \leq y$. Let $u=-x$.

Then

$$
\begin{aligned}
m_{k, n}(\mu)-m_{k, n}(\sigma) & =\left\{(u+1)^{k}+(y+1)^{k}\right\}-\left\{u^{k}+y^{k}\right\} \\
& =k\left(u^{k-1}+y^{k-1}\right)+\left(\begin{array}{l}
k \\
2
\end{array}\right)\left(u^{k-2}+y^{k-2}\right)+\cdots+2 .
\end{aligned}
$$

So again, $m_{k, n}(\mu) \geq m_{k, n}(\sigma)+2$, equality holding only if $x=y=0$. Otherwise, we have

$$
m_{k, n}(\mu) \geq m_{k, n}(\sigma)+2^{k}>m_{k, n}(\sigma)+4 \text { for } k \geq 3 .
$$

REMARK. The proof of the Theorem also shows that $m_{k, n}(\mu)=f_{k, n} \Leftrightarrow \mu=\rho_{n}$. If $\tilde{\sigma} \in S_{n-2}$ we can construct a $\sigma \in S_{n}$ by defining

$$
\begin{aligned}
& \sigma(1)=n ; \sigma(n)=1 \\
& \sigma(i)=\tilde{\sigma}(i-1)+1: 2 \leq i \leq n-1
\end{aligned}
$$

Clearly,

$$
m_{k, n}(\sigma)=2(n-1)^{k}+m_{k, n-2}(\tilde{\sigma})
$$

Proposition 1. For each $n \geq 1 ; m_{1, n}: S_{n} \rightarrow J_{1, n}$ is surjective.

Proof. It is clear by inspection that $m_{1,1} ; m_{1,2}$ are surjective. Let $n \geq 3$. We proceed by induction. Assume $m_{1, n-1}\left(S_{n-1}\right)=J_{1, n-1}$ and $m_{1, n-2}\left(S_{n-2}\right)=J_{1, n-2}$. Then

$$
m_{1, n}\left(S_{n}\right) \supset m_{1, n-1}\left(S_{n-1}\right)=J_{1, n-1}=\left[0, f_{1, n-1}\right] .
$$

Also, from equation (1)

$$
m_{1, n}\left(S_{n}\right) \supset 2(n-1)+m_{1, n-2}\left(S_{n-2}\right)=\left[2(n-1), 2(n-1)+f_{1, n-2}\right]
$$

But $2(n-1)+f_{1, n-2}=f_{1, n}$ and $f_{1, n-1} \geq 2(n-1)$ for $n \geq 3$. So that $m_{1, n}\left(S_{n}\right)=J_{1, n}$. 
LEMMA 2. Let $k \geq 3$. If $\lambda \in S_{n}$ is such that $m_{k, n}(\lambda)=f_{k, n}-2$ then $n$ is even, say $n=2 m$, and $\lambda=\rho_{n} \circ \tau$, where $\tau$ is the transposition $(m m+1)$

Proof. Suppose $\lambda \in S_{n}$ and $m_{k, n}(\lambda)=f_{k, n}-2$. Since $\lambda$ clearly does not equal $\rho_{n}$, the remarks included in the proof of Theorem 1 allow us to conclude that there is a transposition $\tau$ such that if $\mu=\lambda \circ \tau$ then $m_{k, n}(\mu) \geq m_{k, n}(\lambda)+2=f_{k, n}$. But we must have equality, so that $\mu=\rho_{n}$, and for some $i, \lambda(i)=i ; \lambda(i+1)=$ $i+1$. Thus $\lambda=\rho_{n} \circ \tau$ and it follows that $i$ must equal $m$ and $\tau=(m m+1)$.

THEOREM 2. For $k \geq 3 f_{k, n}-4 \notin m_{k, n}\left(S_{n}\right)$

Proof. Suppose there exists a permutation $\sigma \in S_{n}$ such that $m_{k, n}(\sigma)=f_{k, n}-4$. There exists a transposition $\tau_{1}$ of the kind described in Theorem 1. If $\mu_{1}=\sigma \circ \tau_{1}$ then $m_{k, n}\left(\mu_{1}\right) \geq m_{k, n}(\sigma)+2=f_{k, n}-2$. Now equality must hold since we have seen that otherwise, $m_{k, n}\left(\mu_{1}\right)>m_{k, n}(\sigma)+4=f_{k, n}$ which cannot happen. But if $m_{k, n}\left(\mu_{1}\right)=f_{k, n}-2$, then by Lemma $2, n$ is even, say $=2 m$, and

$$
\mu_{1}=\left(\begin{array}{c}
1 \ldots m-1 m m+1 m+2 \ldots 2 m \\
2 m \ldots m+2 m m+1 m-1 \ldots 1
\end{array}\right)
$$

But $\sigma=\mu_{1} \circ \tau_{1}$ and $\sigma(i)=i, \sigma(i+1)=i+1$ for some $i$. This clearly cannot happen so the Theorem is proved.

3. $m_{3, n}\left(S_{n}\right)$ and a Conjecture. In this section we prove:

THEOREM 3. $m_{3, n}\left(S_{n}\right) \supset\left[0, f_{3, n}-112\right] \cap J_{3, n}$ for $n \geq 10$.

Using the available computer facilities (IBM 360-40) $m_{3, n}\left(S_{n}\right)$ was tabulated for $n \leq 9$. This information, plus a few simple calculations is enough to show that $m_{3,10}\left(S_{10}\right) \supset[0,2338] \cap J_{3,10}$; and $m_{3,11}\left(S_{11}\right) \supset[0,3488] \cap J_{3,11}$. A few details on the computations are included for completeness.

From the tabulation it is known that $m_{3,9}\left(S_{9}\right) \supset[0,1488] \cap J_{3,9} \sim\{24\}$, and it is clear that $24 \in m_{3,10}\left(S_{10}\right)$. Thus $m_{3,10}\left(S_{10}\right) \supset[0,1488] \cap J_{3,10}$. Also from equation (1) of $\S 2, m_{3,10}\left(S_{10}\right) \supset 2\left(9^{3}\right)+m_{3,8}\left(S_{8}\right)=1458+m_{3,8}\left(S_{8}\right)$. From the tabulation of $m_{3,8}\left(S_{8}\right)$ it is seen that of the numbers less than 922 , only 24,168 , 734 , and 898 are missing from the set $m_{3,8}\left(S_{8}\right)$. Now $1626=1458+168 \epsilon$ $m_{3,10}\left(S_{10}\right)$ since

$$
1626=9^{3}+8^{3}+7^{3}+2^{3}+2^{3}+2^{3}+2^{3}+2^{3}+1^{3}+1^{3}
$$

and we put

$$
\sigma=\left(\begin{array}{rrrrrrrrrr}
1 & 2 & 3 & 4 & 5 & 6 & 7 & 8 & 9 & 10 \\
10 & 4 & 5 & 2 & 7 & 8 & 6 & 9 & 1 & 3
\end{array}\right)
$$

$2192=1458+734 \in m_{3,10}\left(S_{10}\right)$ since

$$
2192=9^{3}+8^{3}+8^{3}+7^{3}+4^{3}+2^{3}+2^{3}+2^{3}+2^{3}
$$


and we put

$$
\sigma=\left(\begin{array}{rrrrrrrrrr}
1 & 2 & 3 & 4 & 5 & 6 & 7 & 8 & 9 & 10 \\
10 & 9 & 5 & 8 & 3 & 4 & 7 & 6 & 1 & 2
\end{array}\right)
$$

Finally $2356=1458+898 \in m_{3,10}\left(S_{10}\right)$, since

$$
2356=9^{3}+8^{3}+8^{3}+7^{3}+5^{3}+5^{3}+2^{3}+1^{3}+1^{3}
$$

and we put

$$
\sigma=\left(\begin{array}{rrrrrrrrrr}
1 & 2 & 3 & 4 & 5 & 6 & 7 & 8 & 9 & 10 \\
10 & 9 & 8 & 4 & 7 & 5 & 6 & 3 & 1 & 2
\end{array}\right)
$$

Thus $m_{3,11}\left(S_{11}\right) \supset[0,2378] \cap J_{3,11}$ and $m_{3,11}\left(S_{11}\right) \supset 2000+m_{3,9}\left(S_{9}\right)$. But 24 is the only even integer less than 1488 which does not belong to $m_{3,9}\left(S_{9}\right)$, and from the first formula we see that $2024 \in m_{3,11}\left(S_{11}\right)$. So that our calculations are complete.

The Theorem is now proved by induction. We assume that for some $n \geq 11$,

$$
m_{3, n}\left(S_{n}\right)>\left[0, f_{3, n}-112\right] \cap J_{3, n}
$$

and

$$
m_{3, n-1}\left(S_{n-1}\right) \supset\left[0, f_{3, n-1}-112\right] \cap J_{3, n-1} .
$$

Then

$$
m_{3, n+1}\left(S_{n+1}\right) \supset m_{3, n}\left(S_{n}\right) \supset\left[0, f_{3, n}-112\right] \cap J_{3, n}
$$

Also,

$$
m_{3, n+1}\left(S_{n+1}\right) \supset 2 n^{3}+m_{3, n-1}\left(S_{n-1}\right) \supset\left[2 n^{3}, 2 n^{3}+f_{3, n-1}-112\right] \cap J_{3, n+1}
$$

Also, $f_{3, n+1}=2 n^{3}+f_{3, n-1}$. Now, $f_{3, n}-112>2 n^{3}$ for $n \geq 10$, so that combining (1) and (2) it follows that

$$
m_{3, n+1}\left(S_{n+1}\right) \supset\left[0, f_{3, n+1}-112\right] \cap J_{3, n+1}
$$

and the Theorem is proved.

It seems reasonable to make the following conjecture. Let $k \geq 1$. Then there exist two integers $\alpha_{k}, n_{k}$ such that

$$
m_{k, n}\left(S_{n}\right) \supset\left[0, f_{k, n}-\alpha_{k}\right] \cap J_{k, n} \text { for } n \geq n_{k} .
$$

The conjecture would be proved if, for the given $k$, we could find an integer $\alpha_{k}$ such that the statement is true for two successive integers $n, n+1$ with $f_{k, n}>2 n^{k}+\alpha_{k}$.

\section{REFERENCES}

1. J. L. Davison. A Result on Sums of Squares, Canadian Mathematical Bulletin Vol. 18(3), $1975,425-426$.

\footnotetext{
DepartMENT OF MATHEMATICS

LAURENTIAN UNIVERSITY

SudBury, ONTARIO.
} 